\title{
A CASE OF MULTIPLE PROXIMAL JEJUNAL DIVERTICULAE CAUSING INTESTINAL OBSTRUCTION: A VERY RARE PRESENTATION
}

Challapalli Srikanth Reddy11, Battalapalli Srihari Rao², Thalamati Sujana ${ }^{3}$, Shankar Reddy Dudala4, Korchapati Ajay Babu ${ }^{5}$

\section{HOW TO CITE THIS ARTICLE:}

Challapalli Srikanth Reddy, Battalapalli Srihari Rao, Thalamati Sujana, Shankar Reddy Dudala, Korchapati Ajay Babu. "A Case of Multiple Proximal Jejunal Diverticulae Causing Intestinal Obstruction: A very rare Presentation". Journal of Evolution of Medical and Dental Sciences 2015; Vol. 4, Issue 03, January 08;

Page: 469-475, DOI: $10.14260 /$ jemds/2015/68

\begin{abstract}
Diverticulosis of small intestine is an uncommon disease, with variable clinical presentation. Among the small bowel diverticulae, distal jejunal diverticulae form the most common subset. Diverticulosis of proximal jejunum represents an uncommon pathology of the small bowel. The condition is usually asymptomatic and should be taken into consideration in cases of unexplained malabsorption, anemia, chronic abdominal pain or discomfort. Complications such as diverticulitis, perforation, bleeding or intestinal obstruction appear in $10-30 \%$ of the patients increasing morbidity and mortality rates. We herein report a case of a 45 year-old man, who presented at the emergency department with acute abdominal pain, vomiting and abdominal distension. Preoperative radiological examination followed by laparotomy revealed multiple proximal jejunal diverticulae, filled with food, adherent to and compressing upon the terminal ileum, causing intestinal obstruction.
\end{abstract}

KEYWORDS: Multiple Diverticulae, Proximal Jejunum, Compressing upon terminal ileum, Abdominal distension, Intestinal Obstruction.

INTRODUCTION: Diverticulosis of the small intestine is an uncommon disease, with variable clinical presentation. Jejunum is the most commonly involved part of small Intestine, accounting for $85 \%$ of the cases. Proximal jejunum, in turn is very rarely involved by diverticulosis. ${ }^{1}$ The disease is usually asymptomatic and must be taken into consideration in cases of unexplained malabsorption, anemia, chronic abdominal pain or discomfort. Related complications such as diverticulitis, perforation, bleeding or intestinal obstruction appear in $10-30 \%$ of the patients, increasing morbidity and mortality rates. We herein report a case of 45 year-old male presented at the emergency department with acute abdominal pain, vomiting and abdominal distension. Preoperative radiological examination followed by laparotomy revealed multiple proximal jejunal diverticula, filled with food, compressing upon the terminal ileum, causing Intestinal obstruction, due to extrinsic compression.

CASE REPORT: A middle aged male, chronic alcoholic, aged about 45 years presented to the emergency department with the complaints of pain all over the abdomen, 4 episodes of vomiting, abdominal distension and absolute constipation, all within 48 hours prior to his presentation. He had a history of recurrent abdominal pain, fullness after taking food and distension since 3 years, which used to subside upon taking local treatment. He had not received any drugs in the previous 3 months.

On Physical examination, he was febrile with a temperature of $100 \mathrm{~F}$, his pulse rate measuring 110/min and blood pressure measuring 90/70mm of Hg. Abdomen was soft and distended, with increase in the frequency of bowel sounds. Ballooning of rectum was noted on rectal examination. 
Abnormal laboratory findings include leukocytosis $\left(13000 / \mathrm{mm}^{3}\right)$ and hypokalemia (3.3 mmol/l). Serum Amylase and Serum lipase were in the normal range. A plain abdominal X-ray showed multiple air-fluid levels and dilated intestinal loops suggesting intestinal obstruction but with no signs of perforation (Figure 1). Abdominal ultrasonography revealed dilated and hyperactive intestinal loops without any evidence of free intraperitoneal fluid.

The patient was resuscitated with intravenous fluids, nasogastric tube and treated with antibiotics (Ceftriaxone and metronidazole). Emergency surgery was contemplated for managing the acute intestinal obstruction. Intra operatively, 3 large jejunal diverticulae were noted in the mesenteric border of proximal jejunum, most proximal one lying about $20 \mathrm{~cm}$ distal to duodenojejunal flexure. The diverticulae were noted to be enlarged, due to filling up with food material and found to be compressing upon terminal ileum. Adhesions between the terminal ileum and the diverticulae were lysed and resection of the part of jejunum affected by diverticulae was done [Figure 2, 3]. Primary end to end anastamosis of jejunum was done in two layers. Post-operative period was uneventful.

DISCUSSION: Diverticulosis of the small bowel is a rare disease with variable clinical presentations and often incidentally discovered during radiological investigations. The disease was first described by Sommering in 1794 and later by Astley Cooper in 1809. Gordinier and Shil performed the first operation for diverticulae in 1906.1,2 These diverticulae are usually pseudodiverticulae, resulting from a mucosal and submucosal herniation through the muscle layer at places of minor weakness, especially at points where blood vessels penetrate the intestinal wall. ${ }^{2}$ The etiology is unclear.

Krishnamurthy et al, ${ }^{3}$ focused on abnormalities of the smooth muscles or of the myenteric plexus in order to explain intestinal dyskinesia. Kongara et al, ${ }^{4}$ performed manometric studies of the small bowel and described functional abnormalities in patients with small bowel diverticulae. These facts support the hypothesis that irregular intestinal contractions generate increased segmental intraluminal pressure, favoring the diverticula formation through the weakest point of the bowel. A connection between intestinal diverticulosis and rare neuromuscular disorders such as CronkhiteCanada syndrome, ${ }^{5}$ Fabry's disease, Ehlers-Danlos syndrome and systemic sclerosis. Two cases of familiar predisposition have been reported.6,7

Eighty percent of diverticula occur in the jejunum, fifteen percent in the ileum and five percent in both. Isolated jejunal diverticulosis coexists with diverticula of the esophagous (2\%), of the duodenum (26\%) and of the colon (35\%). ${ }^{8}$ Prevalence increases with age with a peak incidence at the sixth and seventh decades with a male predominance. ${ }^{9}$ The size of small bowel diverticulae varies. Diverticula may measure from few millimeters up to more than $3 \mathrm{~cm}$.

The disease is usually silent. Nevertheless, Rodrigez et $\mathrm{al}^{8}$ reviewed the literature and noted symptoms in $29 \%$ of the cases. Many symptoms may be misdiagnosed as dyspepsia or irritable small bowel. Edwards described a symptom triad observed in these patients as 'flatulent dyspepsia' epigastric pain, abdominal discomfort, flatulence one or two hours after meals. ${ }^{10}$ Vague and chronic, mainly post prandial, epigastric cramping pain, bloating or abdominal fullness is usually referred. Anemia due to iron deficiency and megaloblastic anemia have often been reported and commonly attributed to malabsorption, steatorrhoea, and vitamin C deficiency. ${ }^{11}$ Malabsorption could be the result of non-synchronous peristaltic movement of the bowel, the dilation of the diverticula, the stasis 
of the intestinal content and the bacterial overgrowth. ${ }^{11,12}$ Complications such as obstruction, hemorrhage, diverticulitis and perforation occur in $10 \%-30 \%$ of the patients. ${ }^{12}$

Mechanical obstruction can be caused by adhesions or stenosis due to diverticulitis, intussusception at the site of the diverticulum and volvulus of the segment containing the diverticula. In addition, sizable stones enclosed in the diverticula may apply pressure to the adjacent bowel wall or may escape from the diverticulum causing intestinal occlusion. $10-25 \%$ of diverticulae present with pseudo obstruction which occurs as a result of peritonitis (following diverticulitis), perforation, strangulation and incarceration of an enterolith within a diverticulum or related to the bacterial overgrowth and the visceral myopathy or neuropathy. ${ }^{13} \mathrm{~A}$ wide, overloaded with liquid diverticulum may function as a pivot causing volvulus. ${ }^{14}$

Suspicion of jejunal diverticulosis is difficult and often the diagnosis is missed or delayed. Considering that jejunal diverticulosis is asymptomatic for a long time in most of the cases, diagnosis is usually made when the disease becomes symptomatic or complicated. Simple radiographs are not suggestive to make the diagnosis despite the fact that Nobles et al $^{15}$ described a characteristic triad of clinical and radiographic findings of jejunoileal diverticulosis (Abdominal pain, anemia and segmental dilatation in the epigastrium or in the left upper abdomen). But it may be helpful in diagnosing the complications. Barium follow-through study and enteroclysis are more specific although their utility is limited in emergency conditions. ${ }^{16}$ Computed tomography may show focal areas of out-pouching of the mesenteric side of the bowel, localized intestinal wall thickening due to inflammation or edema and other complications. Multi slice CT seems to be promising in diagnosing jejunoileal diverticula and appears more specific than enteroclysis concerning small bowel diseases. ${ }^{17}$ Endoscopy does not identify diverticula but excludes other causes of obstruction or hemorrhage.

Asymptomatic jejunoileal diverticulae do not require surgical intervention. Chronic symptoms with jejunal diverticula can also be treated conservatively and if symptoms are persistent and refractory to treatment then resection is imperative. Complications arising out of diverticulae warrant exploratory laparotomy and resection of involved segment with primary anastamosis.

Jejunoileal diverticulae, compared to diverticulae of the duodenum, have a greater propensity to perforate and develop abscesses, and hence, a more aggressive surgical approach is recommended.18 The extent of the segmental resection depends on the length of the bowel affected by diverticula. If diverticula involve a long intestinal segment, as commonly happens, the resection should be limited to the perforated or inflamed intestinal segment in order to avoid a short bowel syndrome. Other surgical approaches such as the invagination of the diverticula, the primary closure of the perforation and omental patch and the diverticulectomy should be avoided since they present high mortality rates. ${ }^{19}$ Diverticula have a great tendency to recur following segmental resection since the mechanism of diverticula formation (neuropathy, myopathy etc.) still remains. ${ }^{20}$ Enterotomy or segmental resection should be reserved for complicated cases.

In the case reported in this paper, the patient was a chronic alcoholic and had chronic abdominal discomfort or pain, for which he had been taking treatment. The intestinal obstruction was the main symptom of presentation and obviously due to multiple overloaded jejunal diverticulae, compressing upon the terminal ileum, with adhesions, probably due to prior diverticulitis. 
CONCLUSION: Delayed diagnosis of jejunal diverticulae results in increased morbidity and mortality and hence a high degree of suspicion is necessary in patients, presenting with chronic pain abdomen. Radiological investigations and enteroclysis are likely to diagnose diverticulae early and avert the complications. The treatment of choice is surgical excision of the affected jejunal segment. But diverticulae are likely to recur due to persisting underlying cause.

\section{REFERENCES:}

1. Longo WE, Vernava AM: Clinical implications of jejunoileal diverticular disease. Dis Colon Rectum 1992, 35:381-388.

2. Williams R, Davidson DD, Serota AL, Wilson SE: Surgical problems of diverticula of the small bowel. Surg Gynecol Obstet 1981, 152:621-6.

3. Krishnamurthy S, Kelly MM, Rohrmann CA, Schuffler MD: Jejunal diverticulosis. A heterogeneous disorder caused by a variety of abnormalities of smooth muscle or myenteric plexus. Gastrenterol 1983, 85:538-547.

4. Kongara KR, Soffer EE: Intestinal motility in small bowel diverticulosis: a case report and review of the literature. J Clin Gastroenterol 2000, 30:84-6.

5. Cunliffe WJ, Anderson J: Case of Cronkhite-Canada syndrome with associated jejunal diverticulosis. Br Med J 1967, 4:601-2.

6. Koch AD, Schoon EJ: Extensive jejunal diverticulosis in a family, a matter of inheritance? Neth J Med 2007, 65:154-155.

7. Andersen LP, Schjoldager B, Halver B: Jejunal diverticulosis in a family. Scand J Gastroenterol 1988, 23:672-4.

8. Rodriguez HE, Ziaudin MF, Quiros ED, Brown AM, Podbielski FS: Jejunal diverticulosis and gastrointestinal bleeding.

9. Lempinen M, Salmela K, Kemppainen E: Jejunal diverticulosis: a potentially dangerous entity. Scand J Gastroenterol 2004, 39:905-9.

10. Edwards HC: Diverticula of the Small Intestine. B J of Radiology 1949, 22:437-442.

11. Drude RB Jr, Finkelman D, Davis WD, Ferrante WA: Malabsorption in jejunal diverticulosis treated with resection of the diverticula. Dig Dis Sci 1980, 25:802-6.

12. Akhrass R, Yaffe MB, Fischer C, Ponsky J, Shuck JM: Small-bowel diverticulosis: Perceptions and reality. J Am Coll Surg 1997, 184:383-8.

13. Eckhauser FE, Zelenock GB, Freier DT: Acute complications of jejuno-ileal pseudodiverticulosis: Surgical implications and management. Am J Surg 1979, 138:320-323.

14. Chou CK, Mak CW, Hou CC, Chang JM: CT of large small bowel diverticulum. Abd Imaging 1998, 23:132-4.

15. Nobles E: Jejunal diverticula. Arch Surg 1973, 102:372-374.

16. Benya EC, Ghahremani GC, Brosnan JJ: Diverticulitis of the jejunum. Clinical and radiological features. Gastrointest Radiol 1991, 16:24-28.

17. Bitterling H, Rock C, Reiser M: Computed tomography in the diagnosis of inflammatory bowel disease-methodology of MSCT and clinical results. Radiologe 2003, 43:17-25.

18. Chendrasekhar A, Timberlake GA: Perforated jejunal diverticula: an analysis of reported cases. Am Surg 1995, 61:984-988. 


\section{CASE REPORT}

19. Englund R, Jensen M: Acquired diverticulosis of the small intestine: case reports and literature review. Aust N Z J Surg 1986, 56:51-54.

20. Evangelos F, Konstantinos V, StavrosM, Fotis A and Constantinos V, Multiple giant diverticulae of the jejunum causing intestinal obstruction: report of a case and review of the literature.

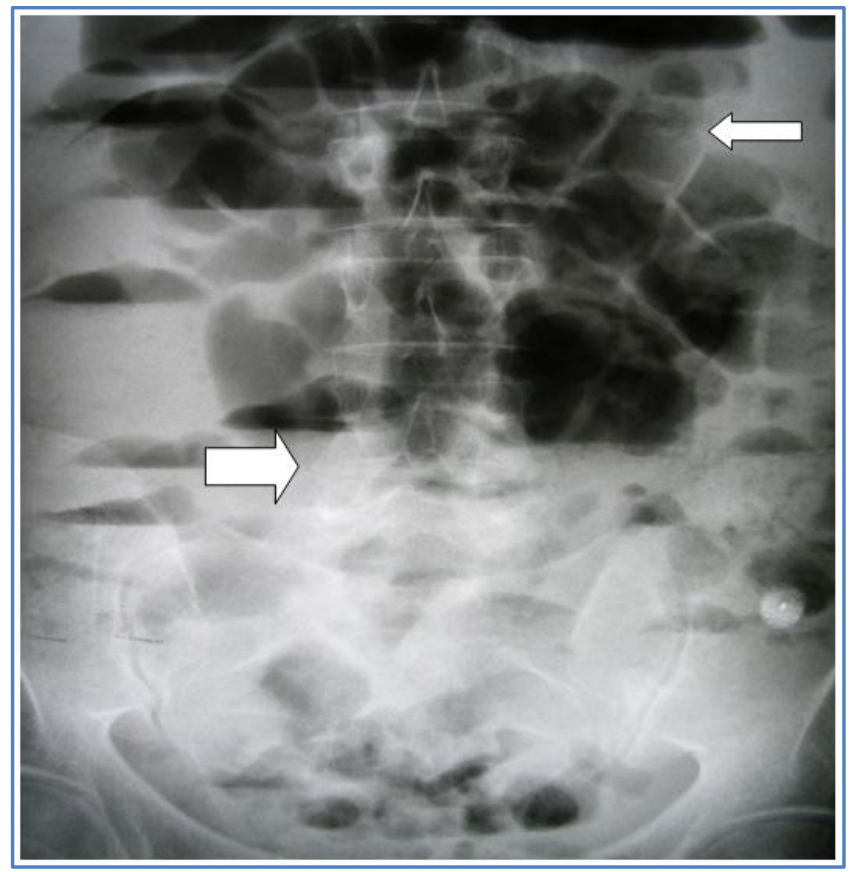

Fig. 1: Plain X Ray Abdomen showing multiple dilated Small Bowel loops \& Air fluid levels

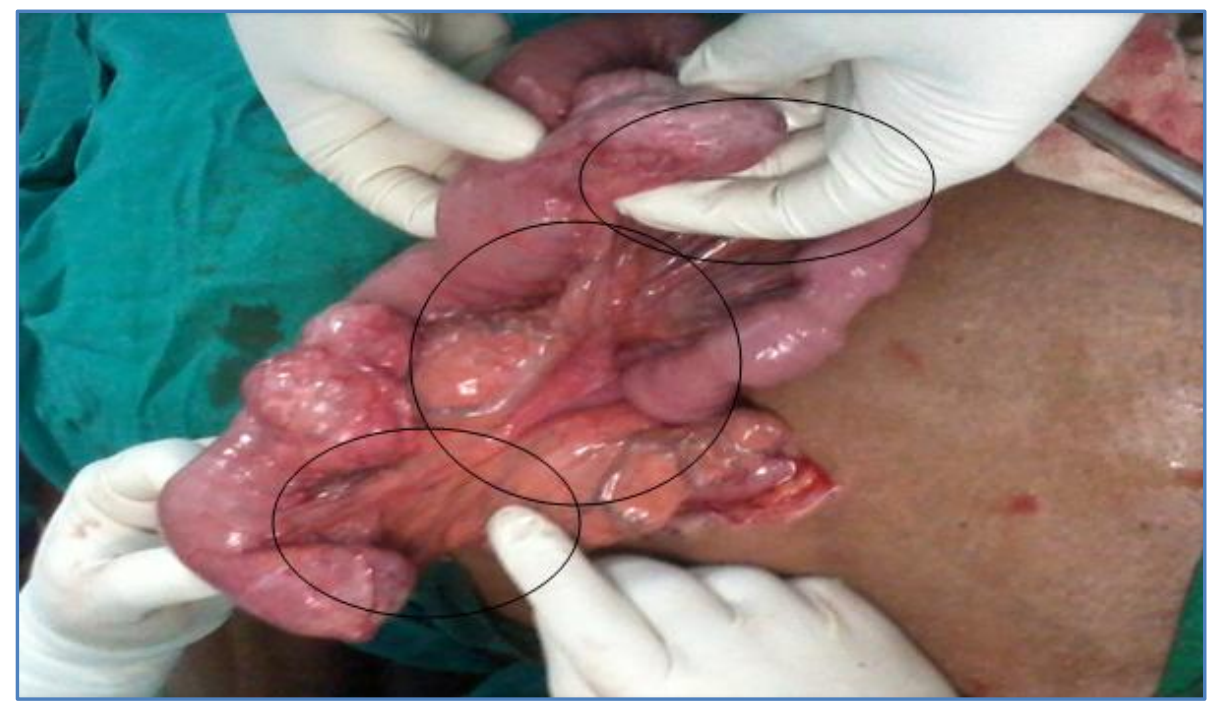

Fig. 2: Intra Operative picture showing multiple Jejunal diverticulae 


\section{CASE REPORT}

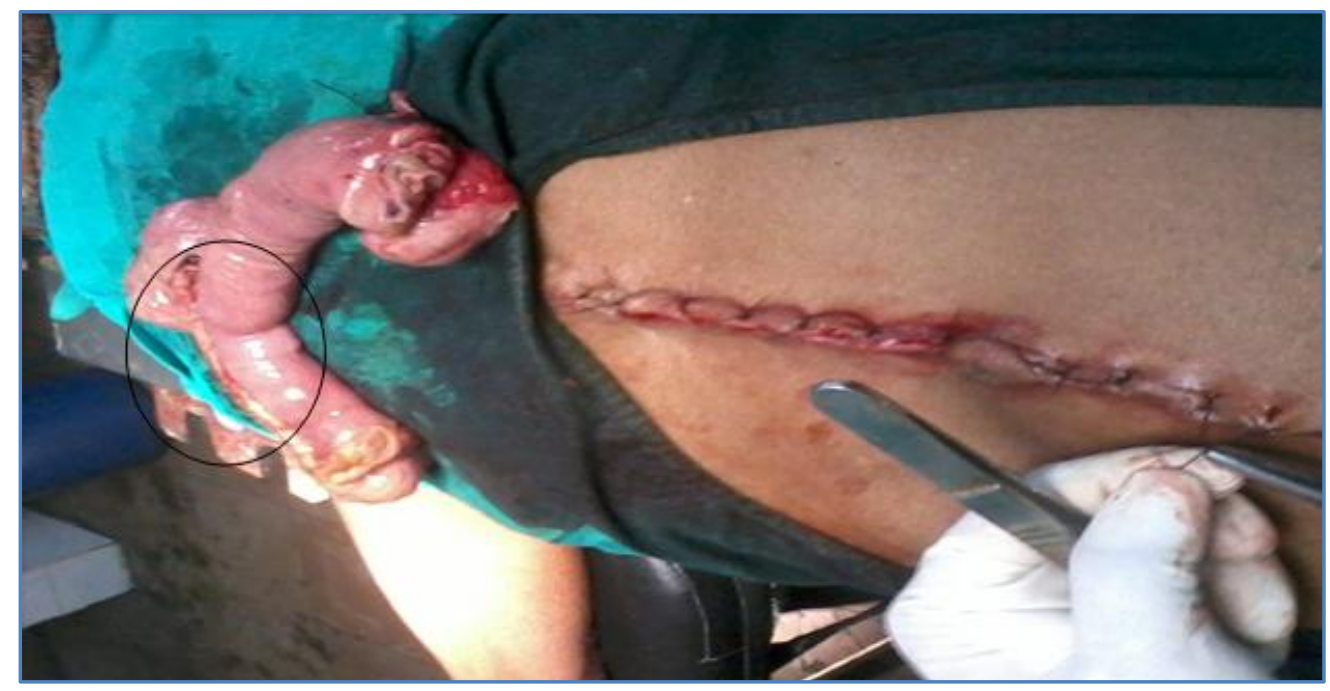

Fig. 3: Picture of the specimen after Bowel Resection

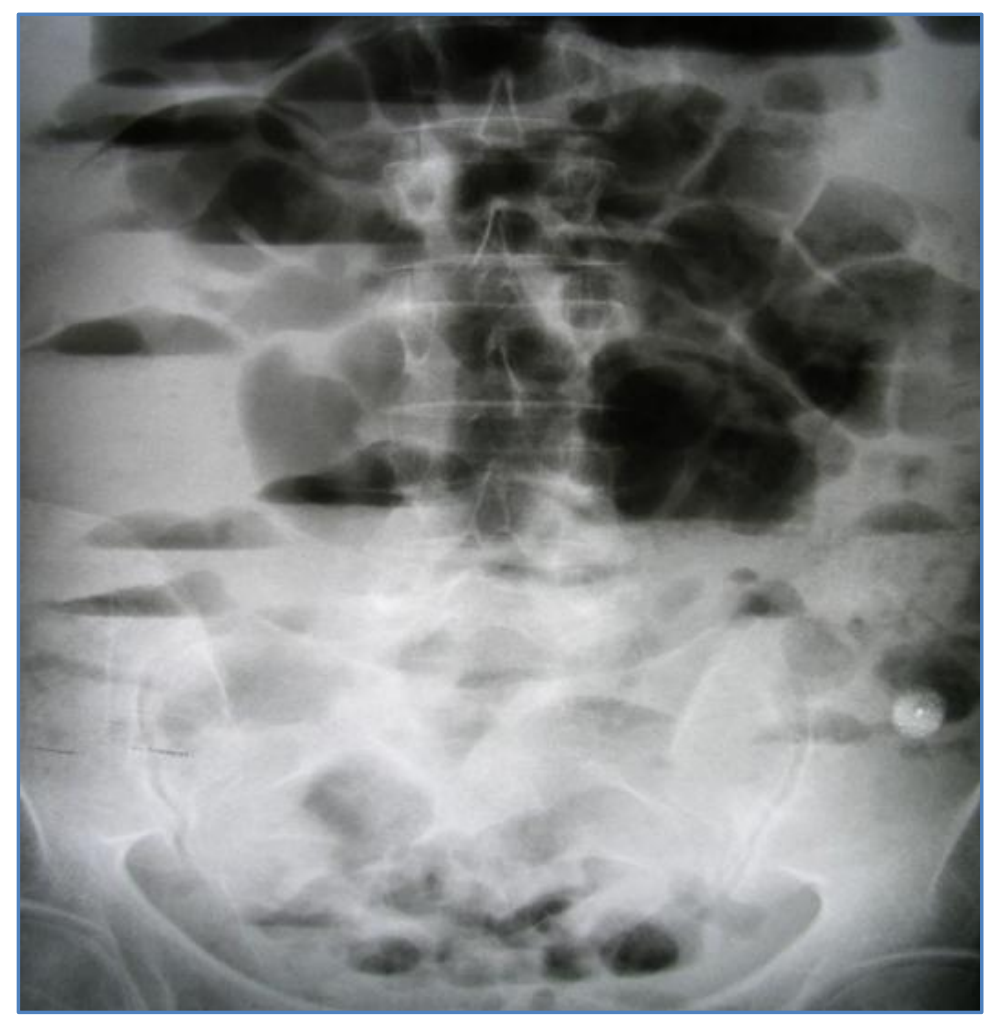

Fig. 4: Pre operative radiograph showing acute intestinal obstruction 


\section{CASE REPORT}

\section{AUTHORS:}

1. Challapalli Srikanth Reddy

2. Battalapalli Srihari Rao

3. Thalamati Sujana

4. Shankar Reddy Dudala

5. Korchapati Ajay Babu

\section{PARTICULARS OF CONTRIBUTORS:}

1. Assistant Professor, Department of General Surgery, SV Medical College, Tirupati.

2. Professor, Department of General Surgery, SV Medical College, Tirupati.

3. Assistant Professor, Department of Obstetrics and Gynaecology, DD Medical College, Tamilnadu.
4. Assistant Professor, Department of Community Medicine, SV Medical College, Tirupati.

5. Internee, SVMC, Tirupati.

\section{NAME ADDRESS EMAIL ID OF THE CORRESPONDING AUTHOR:}

Dr. C. Srikanth Reddy, Assistant Professor, Department of Surgery, S. V. Medical College \& S. V. R. R. G. G. Hospital, Tirupati-517501.

E-mail: csrikanth_ms@yahoo.co.in

Date of Submission: 23/12/2014.

Date of Peer Review: 24/12/2014.

Date of Acceptance: 30/12/2014.

Date of Publishing: 06/01/2015. 